\title{
Débitmètres électromagnétiques
}

\author{
P. Baudet \\ DANFOSS France
}

\section{Principe de mesure}

La mesure se fonde sur la loi de FARADAY qui énonce : une charge électrique $q$ de largeur $L$ se déplaçant à la vitesse $V$ perpendiculairement à un champ magnétique $B$ crée une différence de potentiel induite suivant la formule $U=B . L . V$.

Si l'on applique ce principe au débit d'un fluide conducteur dans une conduite pleine placé dans un champ magnétique on peut déduire qu'un liquide conducteur, se déplaçant dans un champ magnétique, produit une différence de potentiel que l'on peut mesurer entre deux électrodes placées sur la conduite (voir schéma 1).

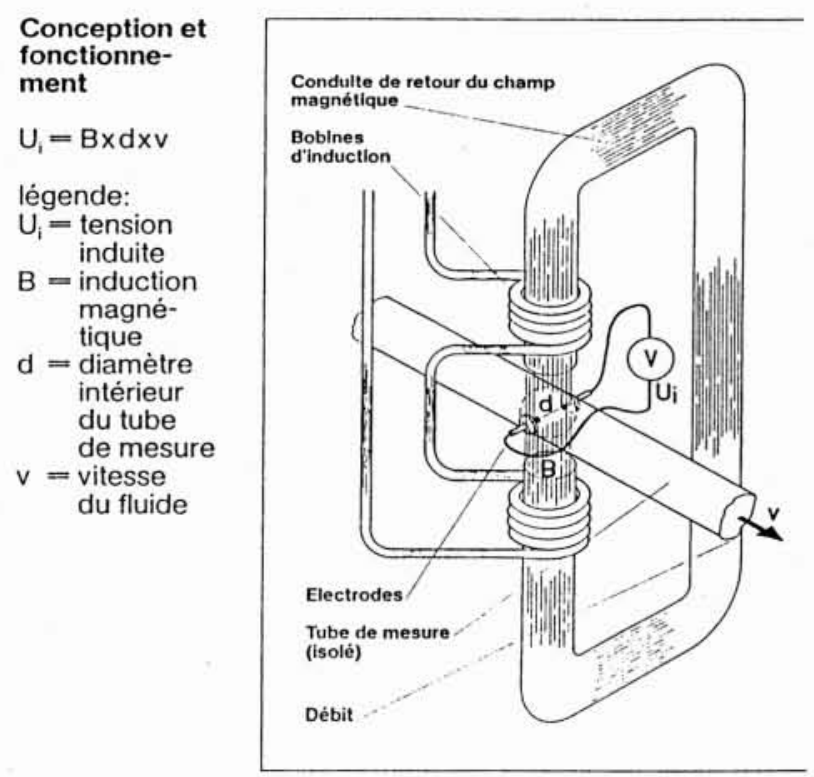

1. Principe de fonctionnement du débitmètre électromagnétique.
Afin de s'affranchir des problèmes de dérive de zéro et de polarisation des électrodes, on applique un champ magnétique continu alterné à une fréquence qui dépend de la précision et du diamètre de la tuyauterie utilisée. Ces commandes sont initiées à partir d'un transmetteur de mesure.

\section{Transmetteurs}

Outre la fonction décrite ci-dessus, le transmetteur (ou convertisseur) est chargé de délivrer des signaux de mesure normalisés (0-20 mA/4-20 mA/impulsions/fréquences) et autres informations complémentaires selon les options proposées. Les récents progrès de l'électronique ont permis d'intégrer des microprocesseurs dans les transmetteurs offrant ainsi de nouvelles performances de calcul, de précision, une meilleure convivialité de mise en œuvre, une meilleure immunité aux bruits électriques, hydrauliques et aux parasites électromagnétiques.

\section{Performances}

Parmi les dernières nouveautés accessibles aujourd'hui sur le marché, on peut citer :

- orientation indifférente des modules d'affichage indépendamment de la position du capteur (en montage compact) ;

- mesure bidirectionnelle avec comptages associés et signalisation par relais ;

- coupure faible débit (pour ne pas mesurer les fuites et effets de pression statique avec relais de signalisation...); - détection tuyauterie vide (ou basse conductivité);

- ajustement automatique de plage de mesure en fonction de la vitesse de passage du fluide ;

- signaux de sortie différents (courant, fréquence, impulsion de largeur variable);

- précision garantie de $0,25 \%$ de la mesure avec répétabilité meilleure que $0,1 \%$;

\section{Electromagnetic flowmeters}

The last developments of electromagnetic flowmeters led to better performances. But some precautions must be taken when setting up devices in order to guarantee their performances. Gauging at the factory in accredited and interconnected laboratories is now possible, following european rules. The authors show the advantages of the SENSORPROM ${ }^{\otimes}$ from Danfoss. 
- nombreux réglages accessibles en langage clair par mesure (type de sortie, débit max., unité, choix des affichages) ;

- affichage et mémorisation des derniers défauts ;

- conformité aux nouvelles normes européennes sur émission et immunité aux bruits électriques EN50081-1/2 et EN50082-1/-2 ;

- meilleure immunité aux bruits de mesure grâce au filtre d'entrée breveté DANFOSS :

- aide au diagnostic et à la maintenance par affichage de code de défaut, modification de la fréquence de sortie (pour tester les appareils externes associés), forçage du relais de signalisation ;

- mémorisation des paramètres dans une mémoire non volatile EEPROM (SENSORPROM ${ }^{\circledR}$ de DANFOSS) associée au capteur de mesure permettant de charger l'électronique sans refaire le paramétrage et démarrer une installation en moins de 2 minutes par simple mise sous tension.

Dans un futur proche les capteurs pourront être reliés à un bus de terrain que les fabricants des capteurs souhaitent le plus universel possible.

\section{Installation}

Des précautions d'installation élémentaires bien que non contraignantes restent nécessaires pour la bonne utilisation d'un débitmètre électromagnétique, on peut citer :

- garantir le remplissage de la tuyauterie ;

- éviter par un montage astucieux les risques d'encrassement ou de dépôt sur les électrodes :

- aménager des longueurs droites sans perturbation en amont et en aval du débitmètre (5D amont et 3D aval sont des valeurs généralement admises) pour assurer les précisions spécifiées. Il est à remarquer que les perturbations amont amènent une dérive de précision bien qu'elles soient plus importantes que les perturbations aval.

\section{5. Étalonnage}

Les débitmètres sont tous étalonnés sur des bancs agréés par des organismes indépendants officiels. Les différents « laboratoires » s'échangent mutuellement leurs étalons car c'est le seul moyen réel efficace d'intercontrôler les appareils.

Des normes européennes d'essais vont être mises en place afin que les spécifications annoncées par les constructeurs soient réellement comparables (propositions ISO/DP9104 et DIN 19200 par exemple). Les moyens de contrôles périodiques de l'électronique sont simples à mettre en place avec des simulateurs de signaux, quelques mesures sont également envisageables sur les tubes de mesure.

Le réglage absolu serait le passage sur banc d'essai mais ceci est coûteux et ne peut être fait sans démontage du capteur. Mais les débitmètres électromagnétiques ne possédant aucune pièce mécanique en mouvement ni obstacle dans le flux susceptible de s'user, on peut supposer une très grande stabilité côté capteur dans cette technologie.

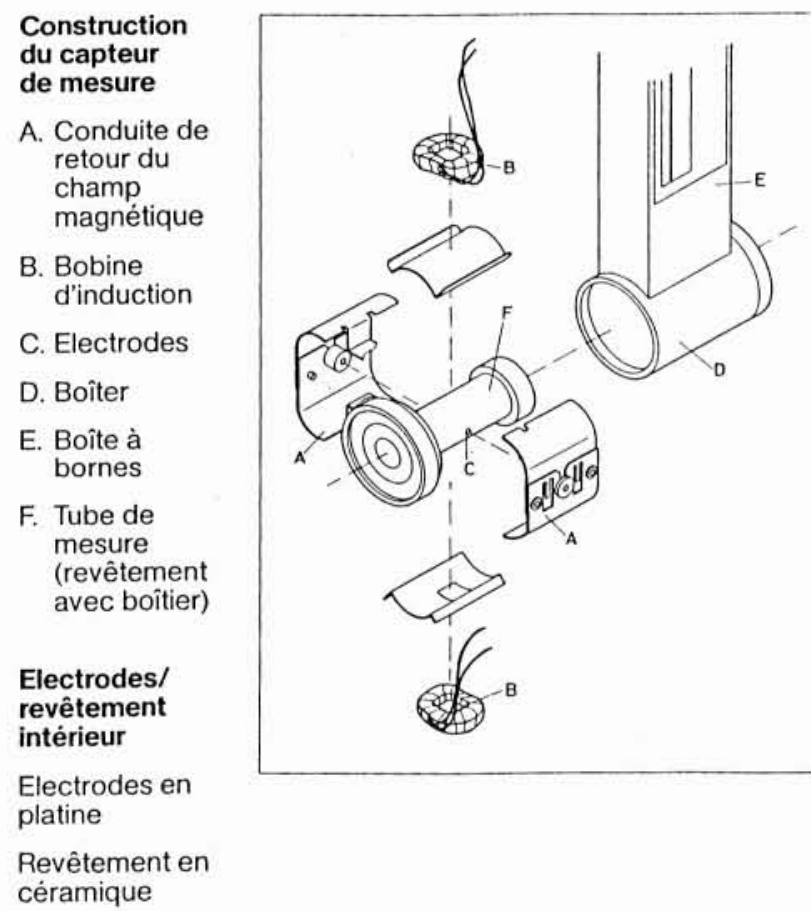

2. Construction du capteur de mesure. 